\title{
Beyond virtual cultural tourism: history-living experiences with cinematic virtual reality
}

Marasco, Alessandra ${ }^{a}$

${ }^{a}$ Institute for Research on Innovation and Services for Development (IRISS) - National Research Council (CNR), Italy. a.marasco@iriss.cnr.it

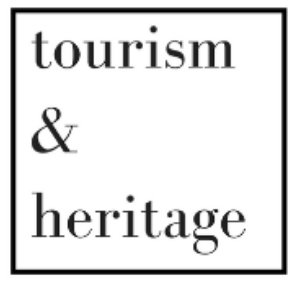

J O U R N A L

ART ICLE INFO

Article history:

Received 27 April 2020

Accepted 21May 2020

Published 25 May 2020

Keywords:

Cinematic Virtual Reality; Cultural heritage; Emotions; Storytelling; Tourism.

\section{ABS T RACT}

Virtual reality (VR) offers unprecedented opportunities for creating cultural tourism experiences that tell visitors emotionally engaging stories about the past. This paper focuses on cinematic virtual reality, the latest frontier of immersive storytelling rivalling feature films, which can immerse users into 360-degree films making them feel like living the story. Through a qualitative analysis of five projects, it explores how this new media form has been applied for cultural heritage storytelling and analyses the reactions of users to the historyliving experiences with an emphasis on their emotional responses. Based on the findings of the analysis, implications are discussed for the design of compelling VR experiences in cultural tourism.

DOI: 10.1344/THJ.2020.2.1

\section{INTRODUCTION}

Tourism and heritage have been profoundly impacted by Virtual Reality (VR) and its potential to facilitate access to cultural sites and resources and to enhance visitors' experiences in-situ as well as ex-situ (Bekele et al., 2018; Gavalas et al., 2020; Guttentag, 2010). VR experiences are characterized by physical immersion and psychological sense of presence of users in a virtual environment (Gutiérrez et al., 2008), which can positively influence their enjoyment, attitude and behavioral intentions in the tourism context (Tussyadiah et al., 2018).

VR enables people to travel without boundaries using wearable devices (e.g., Oculus Rift, Google Cardboard, Samsung Gear VR, HTC's Vive), exploring and enjoying attractions and destinations as a prelude or substitute to visitation, an extension of previous experiences, as educational and/or entertainment experiences (Kim et al., 2020). In the last years, VR tours have been created by major cultural heritage institutions, including the Smithsonian National Museum of Natural History, the Palace of Versailles, the Vatican Museums, The State Hermitage Museum. Destinations have embraced VR for cultural heritage enhancement and dissemination projects, such as the Korean Heritage project developed in cooperation with Google Arts \& Culture (Min-sik, 2018). Several museums, galleries and 
heritage sites worldwide can be explored through Google VR Expeditions that are specifically targeted for students' educational experiences. Further, the main VR platforms offer a wide range of travel experiences and virtual tours of historical sites and cities by specialized virtual trip companies and filmmakers, such as Escape Now: The Icons that feature whistlestop virtual tours of iconic destinations. Champion (2019) notes that VR tourism can move beyond realistic recreations of actual sites by bringing the past into the present, for example through experiences that tell stories and contested histories from unexplored perspectives.

In this direction, storytelling, traditionally used by cultural institutions to engage audiences, emerges as a key area of VR application. In the last years, many studies and projects have explored the potential and complexities involved into the design of immersive storytelling experiences that can activate deeper personal and emotional connections with cultural heritage (Perry et al., 2017; Pietroni, 2018; Roussou, 2008; Schofield et al., 2018). The recent development of VR systems and devices is opening unprecedented opportunities in this regard providing new media forms to tell engaging narratives, like 360-degree videos and cinematic VR (Argyriou et al., 2020; Rizvic et al., 2020).

Cinematic VR (cVR) identifies experiences delivered through a VR device with "media fidelity approaches found in feature film" (Mateer, 2017, p. 15). While an established definition is still lacking due to the infancy of the medium (Kubitzek, 2019), it refers to a type of experience that immerses users into a narrative unfolding in a linear way (as in traditional movies) within a 360-degree environment (Reyes, 2018). cVR contents can include live action captured by a video camera system as well as computer generated imagery (CGI), created with filmmaking techniques. In these experiences, scenes and sounds are perceived as completely real by viewers (Jaunt, 2018), making them feel like being immersed into a film and physically present in the scene (Ding et al., 2018). For this reason, the term storyliving is often used in relation to these experiences (Jones, 2017). In the last years, this media form has found an increasing application in the fields of journalism, advertising, travel, sports. Major film festivals have created special awards for documentary and fiction cVR films and contents can be experienced through VR platforms, such as Oculus Video, Samsung Video, Within.

While not being widely used due to budget considerations (Schofield et al., 2018), cVR is considered a promising new media form for immersive storytelling in cultural heritage settings that deserves further exploration given its potential for a better sense of presence, higher degree of photorealism and more elaborated scenes and visual effects compared to other approaches (Argyriou et al., 2020; Schofield et al., 2018). However, this is an emerging area and there is a lack of studies to understand how the story living power of cVR can be used to create compelling historyliving experiences. This paper aims to provide a contribution in this direction through an exploratory, qualitative analysis of projects using cVR for cultural storytelling, with an emphasis on their potential for creating emotionally engaging experiences.

\section{OBJECTIVES}

The general objective of this paper is to contribute knowledge for the design of meaningful and engaging VR experiences in cultural tourism, focusing on the latest frontier of immersive storytelling technologies. To this end, it investigates how cVR has been employed in this context and how users perceived these experiences. Specifically, this study performs an exploratory qualitative analysis of five cVR projects considering the three key dimensions of storytelling (Weissenfeld et al., 2017): notably storytellers' aims and motivations, narrative formats and story-receivers' reactions. 


\section{STATE OF THE ART}

\subsection{Immersive storytelling for experiencing cultural heritage}

Storytelling is considered to be the "real work" of museums and other cultural heritage institutions (Bedford, 2001) and is widely adopted to engage different kinds of audiences and facilitate their learning processes through the universal communicative power of stories:

Stories are the most fundamental way we learn. They have a beginning, a middle, and an end. They teach without preaching, encouraging both personal reflection and public discussion. Stories inspire wonder and awe; they allow a listener to imagine another time and place, to find the universal in the particular, and to feel empathy for others. They preserve individual and collective memory and speak to both the adult and the child. (Bedford, 2001, p. 33)

VR technologies are considered to be the most effective solutions for serving the purpose of storytelling in this context (Carrozzino \& Bergamasco, 2010) and are increasingly integrated into contemporary approaches to cultural heritage communication (Bozzelli et al., 2019; Schofield et al., 2018). Palombini (2012) noted that these technologies can amplify the potential of storytelling in this context by two main factors: immersivity, which enhances the emotional impact of virtual representations, and interactivity, which relates to the possibilities of user-computer and user-narrative interaction as well as usercommunity interaction in virtual worlds. More recently, he stressed that the key challenge in digital cultural storytelling, i.e. to harmonize narrative freedom and historical truth, is heightened by the immersivity power of VR, which may induce users in confusing the sensorial quality of the representation with the exactness of the represented information (Palombini, 2017).

A growing research body has explored the challenges for the design of immersive heritage experiences by combining accurate representation with engaging presentation through storytelling (Roussou, 2002). Storytelling is emphasized as a trigger of interest, empathy and imagination of visitors experiencing virtual cultural environments and the importance of remarkable and unforgettable human characters is highlighted for the development of an engaging narrative (Roussou, 2008). In particular, according to Roussou (2008) the challenge is to design the experience "formula" where characters tie into narratives that trigger users' imagination more than just visual sense, producing memorable experiences that successfully 'suspend disbelief' through the narrative engagement. Pietroni (2019) has highlighted the important role of narration as a coherent harmony of texts, recitation style, music and sounds, visual mood, camera movements, rhythm in creating a bridge between past and present through museum objects and arousing emotions in the experience. She also noted that narration still represents the weakest link in the design of immersive experiences, since it rarely follows the precise rules from the original domains of cinema and theather. In the ArkaeVision project, Bozzelli et al. (2019) applied a user-centric approach to create a more engaging user experience with an integrated system that includes elements of digital fiction and storytelling. In their project, the virtual scenarios and characters' features are designed to fuel users' immersion and emotional involvement, which in turn contributed to generate motivation and to activate the learning process more quickly. In relation to the same project, Pagano et al. (2020) have showed how the integration of scenography, art and traditional filmic direction for representing the atmosphere of the past in the digital reproduction generated a strong emotional involvement that impacted users' attention and memorability of the experience. 
Discussing the role of storytelling in virtual museums, Sylaiou \& Dafiotis (2020) draw attention to the importance of emotions as "fulcrums for engaged learning" and for rethinking museums as affective spaces in dialogue with their audiences. Also the research carried out within the EU-funded project EMOTIVE (Emotive virtual cultural experiences through personalized storytelling) explored how to design digital heritage experiences drawing on the power of "emotive storytelling" (Perry et al., 2017). This research stressed the need to address emotions more explicitly in the design process and investigated methods to design drama-based affective narratives that are able to generate strong emotional engagement with the story, empathy with the characters, and connection with the sites (Roussou et al., 2017). The main design indications for emotional cultural experiences identified by this project are (Katifori et al., 2018; Perry et al., 2017): guiding visitors through a story in the present that makes them explore concepts of the past, creating stories that revolve around people and not exhibits, and supporting interaction between characters and real visitors. This approach is also exemplified in the project Becoming Vincent (Calvi \& Hover, 2018), aimed to create an emotionally engaging virtual experience based on a narrative concept of Van Gogh's younger years. This project used the VR Oculus Rift system for creating an encounter to emotionally immerse the user into the painter's life, making him become the artist himself and engage into a deep experience of the painter's heritage. According to Pujol-Tost (2018) there is still a lack of its systematic evaluation of virtual heritage experiences making it difficult to assess the effectiveness of these narrative solutions beyond the usability/user experience level, despite the emphasis on users' emotional involvement.

Most recent studies in this area have focused on the use of new media forms, such as 360degree videos, for creating engaging experiential solutions for cultural heritage communication and education. The pioneristic work by Demetrescu et al. (2016) has showed how cultural storytelling for enhancing the understanding of Roman archaeological sites integrated filmmaking approaches through the use of mixed reality where actors filmed on a virtual set in front of a green screen are integrated with 3D computer-generated environments. In the projects La Villa Livia Reloaded created for the Roman National Museum of Diocleziano's Thermal Baths and Virtual Museum of the Tiber Valley, the visitors can interact with the virtual environment and attend dialogues between the ancient characters living in the roman sites. Rizvic et al. (2020) have discussed how storytelling methods and tools need to be adapted to virtual environments and 360-degree videos to tell stories with emotional and emphatic content that users will find educational and entertaining. Argyriou et al. (2020) have proposed and applied a design methodology for 360-degree video-based VR cultural tours, based on two complementary layers: the experience design layer, covering the design of the narrative, the virtual scenes and actors/narrator's role; the interaction design layer, related to the navigation in the virtual world and the development of gamified techniques for users' engagement. The new field of immersive experiences introduced with cVR is highlighted as an avenue to be explored for the design of engaging storytelling for historical communication (Argyriou et al., 2020; Schofield et al., 2018).

\section{$3.2 \mathrm{cVR}$ as a media solution for immersive storytelling experiences}

As noted by Schofield et al. (2018), VR is often used as a catchall term that encompasses two very different approaches for immersive experiences in cultural heritage settings: the first uses real-time 3D computer graphics to create fully interactive experiences, where viewers can potentially move around scenes, interact with objects and explore at their own pace; the second, commonly termed as cVR, refers to high quality, pre-rendered 360-degree 
videos (live action captured by a series of cameras or CGI) with limited interactivity compared to the first 'game' like approach. In cVR, interactivity with the virtual environment is generally limited to the choice of the angle from which to view the scene as the narrative plays out (Dooley, 2017).

Research on cVR has addressed the need to establish an appropriate grammar for narration with this medium (e.g., Dooley, 2017; Mateer, 2017; Gödde et al., 2018) and to assess users' experience (Pillai \& Verma, 2019; Reyes, 2018) with an emphasis on their emotional responses (Ding et al., 2018; Shin, 2018). Some studies have noted that cVR is not an extension of filmmaking but a new medium with its own grammar (Mateer, 2017; Jones, 2017). Mateer (2017) discussed how existing directing techniques for narrative transportation can be adapted to achieve suspension of disbelief and presence in cVR. Gödde et al. (2018) have stressed the need to carefully consider the role that the viewers play in the scenes, since their sense of presence is significantly influenced by being only an observer or being part of the scene. Reyes (2018) proposed a methodology for assessing user experience dimensions, including agency (usability, autonomy, effectance), immersion, and transformation aspects (enjoyment, affect, desire to repeat the experience) and the level of physical discomfort. In her study, the evaluation of immersion includes both the perceptual immersion (realism of the virtual environment, presence, flow) and narrative immersion (understanding of the story, believability, role identification, curiosity and suspense, aesthetic pleasantness). Pillai \& Verma (2019) have examined how narrative immersion is experienced in an emotional drama, specifically designed for VR cinema, by exploring the 'experiential fidelity', i.e. how close the user's experience is compared to the storyteller's intended experience.

Research has explicitly considered the emotional effects of cVR experiences, also in relation to specific narrative dimensions, since their design can be purposefully aimed to evoke the desired emotional response and create viewers' engagement (Cho et al., 2016; Kubitzek, 2019). Cho et al. (2016) investigated different stimuli for eliciting users' emotions in immersive narratives based on the manipulation of viewer's space and perceived role in it, showing that users reacted with high emotional intensity and felt more immersed in the story when acknowledged as being a character in the film and being given eye contact from actors. On the basis of emotional gratification theory in media use, Ding et al. (2018) have demonstrated the stronger emotional effect of CVR than the traditional 2D film in terms of both subjective experience and objective physiological reaction. Their study found users' emotional reaction to be stronger in relation to four emotions in particular: excitement, nervousness, hostility and jitteriness. It also suggested specific factors in the cVR experience that determine a stronger emotional effect: the higher degree of presence in the scenes, the wide $360^{\circ}$ and first-person viewpoint in the VR environment and the novelty of the medium. According to Jones $(2017,2019)$, this experiential medium can elicit more opportunities for empathy, through stories that develop a belief in users they are "walking in someone else's shoes". Shin (2018) has provided empirical evidence supporting the interaction dynamics of presence, embodiment and empathy in immersive VR storytelling experiences, which then trigger user engagement. This study identified a two-tiered process of VR experience, according to which most viewers experience a sense of presence at first, which then triggers responses that involve the emotional states of empathy, compassion, and embodiment. Based on the appraisal theory of emotion, Durnell (2018) explored emotional reactions of experiencing the Syrian refugee crisis in the VR film Clouds Over Sidra. The analysis revealed that this immersive experience provoked a range of emotional reactions including feelings of sadness, grief and anger, increased empathy and intentions to act related to the crisis.

Research is emerging that addresses the design of storytelling experiences created with cVR in cultural heritage settings. An experimentation of the use of this medium for the promotion of cultural heritage sites at a tourism destination has showed its potential to tell 
living histories about heritage (Del Verme, 2019) and to emotionally involve visitors in the virtual experience (Marasco, 2017; Marasco et al., 2018). Selmanovic et al. (2018) have used this approach to VR storytelling for preservation of intangible cultural heritage, highlighting its effectiveness in creating a close and personal connection with the tradition by transferring feelings and emotions through the virtual and the real-world environments. Montagud et al. (2020) have addressed the needs and challenges for making these experiences accessible regardless of the capacities of the users, their age, language and/or other specific impairments. However, many issues need to be further explored to inform the design of storyliving experiences in this context, including their applicability to different cultural aims/contexts and narrative formats, the viewers' cognitive, affective and conative responses, the experiential elements that trigger these reactions.

\section{AN EXPLORATORY ANALYSIS OF CULTURAL cVR EXPERIENCES}

\subsection{Method}

Given the lack of previous research specifically investigating cVR experiences related to cultural heritage, an exploratory study has been designed to obtain an initial understanding of how cVR is used for storytelling in this context and what reactions these experiences produce in the viewers, with particular attention to their emotional responses. Specifically, the study is set to examine the three main dimensions of storytelling (Weissenfeld et al., 2017): 1) storytellers (qualification, aims, motivations); 2) narratives (contents, format, technical approaches used); 3) story-receivers (reactions of the viewers in terms thoughts, emotions, feelings, behavior change).

To this end, the research design has been articulated into the three main steps of identification of relevant cases (cVR contents), data collection and data analysis. For the first step, a search has been conducted for relevant projects in literature, online video channels (Youtube and Vimeo) and platforms for VR contents (Oculus Video, Samsung Video, Within, VeeVR). From this search, 19 projects have been identified, including a range of different contents/subjects with a varying level of focus on cultural heritage communication. From this list, a selection was made of projects with an explicit cultural purpose that can be freely accessed online, fully experienced with VR headsets and that registered comments by viewers online. The final set of cases includes five projects created in the period 2016-2019 by professional filmmakers in collaboration with cultural institutions with different aims, narrative formats, technological approaches.

For the analysis of these cases, a qualitative approach has been used based on the analysis of information from producers/creators available online (e.g. interviews, information provided on official websites and in specialized resources) and user generated contents represented by the spontaneous public comments posted online (Youtube, VR platforms, Facebook). Douiji et al. (2016) note that YouTube comments present a huge potential for text-based emotion detection, since they relate to opinions, stories and emotions. In this study a knowledge-based detection approach has been used for the explorative analysis, as reported in Aman \& Szpakowicz (2007) and Douiji et al. (2016).

Procedurally, all the comments were identified, downloaded, anonymized and translated into English when in foreign languages. A total of 1604 comments were read multiple times for familiarizing and then analyzed through manual coding in order to examine alignment with themes and experience dimensions derived from the literature as well as investigate new emergent ones. For the qualitative content analysis of user generated data, an emotion annotation task was performed based on the procedure proposed by Aman \& Szpakowicz (2007) to identify expressions of emotions in the texts in relation to the six basic emotion 
categories proposed by Ekman (1992): happiness, sadness, anger, disgust, surprise and fear. To cope with the limitations associated to subjective judgement in this procedure, the text was also analyzed through the publicly available service IBM Watson Developer Cloud Tone Analyser that detects joy, fear, sadness, anger, analytical, confident and tentative tones in texts.

\subsection{Findings}

The cVR projects considered in the analysis widely vary in terms of creators' cultural aims and motivations, featured subjects and typology of cultural heritage sites/objects, narrative formats, approaches for video production. They include short VR films produced by cultural institutions to provide innovative experiences online and onsite as well as documentaries produced by public TV broadcasters and for VR platforms. Contents' durations range from short clips of 3-4 minutes to projects of approximately 10 minutes. Narrative formats include both documentaries and fictional narratives with an historical specific background. Some projects feature only live action footage, others include live action combined with CGI and CGI solely. Overall, the majority of viewers' comments indicate that these projects are considered successful ("great work", "well done!", "great job!", "the best VR experience ever").

The first project entitled Fossil Hunting in the Gobi 360 ${ }^{\circ 1}$ (Drakeford Films, 2016) was produced by the American Museum of Natural History (AMNH) as an episode of its educational web series Shelf Life. This series is aimed "taking the lids off" some of the most incredible specimens and artifacts of its huge collection that are not actually visible to the audience onsite. This experience is a 3-minute-long time travel that transports viewers back in the 1920s, the time of the expedition of the legendary AMNH paleontologist Roy Chapman Andrews, who inspired the character of Indiana Jones. The travel begins across the sands of the Mongolian desert where the viewer can follow the explorer and his team in the discovery of a burial ground of dinosaur and early mammal fossils and it ends inside the museum at contemporary times where the Curator of the Division of Paleontology explains in first-person how the fossils discovered by Andrews are studied today. In the experience, the fossils are highlighted as the link between the protagonist of the epic adventures of the past and the scientific research in the present. In the first part the narrator (voice over) tells the story of the adventurous journey in the inhospitable desert organized by the "flamboyant" explorer while the viewer is immersed into a 100-year-old expedition footage. To reproduce the authentic virtual expedition environment, the creators used archival photos and film reels from the museum library, while live-action shoots at the museum are used for the last part of the experience. With this project, the museum started to explore the use of this new medium to tell other "genuinely exciting" stories behind the many objects of its collection, as the producer explained, "I honestly feel like there are endless stories from our collection we could tell this way" (Brownlee, 2016).

The analysis of comments posted by viewers highlights surprise generated by the perceived novelty of the experience ("I didn't know YouTube provided this kind of experience until now!") as well as the discovery of the innovative power of the technology ("These 360 videos are seriously the coolest thing I've ever seen... crazy technology has come so far"). The quality and aesthetic pleasentness of the video was an element raised by the comments, as for example this expert viewer noted:

\footnotetext{
${ }^{1}$ https://www.amnh.org/shelf-life/fossil-hunting-in-the-gobi-360
} 
"When Film-makers address Cultural History the result can be a revelation to viewers. That said, as someone who works in film and compositing, I personally find the work shown in the above clip aesthetically unattractive. To be specific, the elements jar because of poor clip stabilisation; irregular grading, mismatching of scale between still and motion sources, and distortion as a result of the source media, and the software employed. The combined effect of these elements works to distract my attention from the subject matter, rather than hold my attention."

The experience is perceived as an enjoyable, "fun watch" by viewers, making them also think to real encounters with fossils: "It's a pity they are never find near to where people live so members of the public could have a look".

Another VR time travel is offered by the 10-minute-long VR documentary Gladiators in the Colosseum $360 V R^{2}$ (Faber Courtial, 2017) produced by German public broadcaster ZDF for the series of documentaries Terra X. This experience teleports viewers into the year 80 A.D. to relive the lives of gladiators as part of the special historical series "A day in ..." that aims to provide an unusual and exciting journey into the everyday life of past eras. With this fully immersive project, the broadcaster wanted to set new quality standards for this VR storytelling format. In this project, footage of real actors is combined with CGI to create an accurate and photorealistic digital reconstruction of Rome in collaboration with historians and archaeologists. In this setting, viewers can experience ancient gladiator fights in extreme close-up from within the scenes. The narration (voiceover by a famous actor) emphasizes the role of gladiators as the protagonists of a cruel entertainment industry that attracted large audiences. The climax of the VR documentary is reached at the end, when viewers can witness the bloody conclusion of a gladiator fight close to Emperor Titus and surrounded by 50,000 other spectators.

Also in this case viewers were struck by the power of the technology ("Real madness where the new technology goes!") and many commented the specific 360-degree immersive view ("Great video! The $360^{\circ}$ view actually makes sense"; "Be sure to watch the hammer video with VR device !!!”). Surprise emerges from users' comments in general ("Mind absolutely blown"; "Absolutely stunning!") and emotional arousal for the possibility of history living offered by the technology: "I'm really excited to see what will be possible when this technology develops its full potential. You could easily relive history from the living room." Further, sense of presence in the virtual environment is reported ("Really strong. Feels like I'm in the Colosseum myself. You have to have experience it yourself :)").

Some comments provide indications on its educational use and the positive emotional reactions elicited by the video in this context of fruition: "impressive video, my latin students were thrilled!"; "My Latin teacher showed us the video today and everyone even clapped because they were so excited! Great video, really!". High positive emotions emerge in relation to the spectacularity of the experience and the fight scenes ("I've watched this video so many times! It's amazing! Hollywood movie makers should be forced to study this video intensively before being allowed to add a gladiator scene to their movie $\left({ }^{\wedge}{ }^{\circ}\right)$ "), whereas disappointment and sadness are reported with regard to subject and message of the narration: "It is incomprehensible how such a sophisticated civilization could be so barbaric..."; "Nowhere in the world has so much blood been shed, should I shed tears now, the culture comes from Rome, which also includes entertainment..."; "One of the best things I ever seen! But so sad at the same time".

\footnotetext{
${ }^{2}$ https://faber-courtial.de/portfolio/gladiatoren-vr/
} 
Intense emotional feelings are also reported in general ("I was scared at the beginning"; "pure goosebumps"), and in relation to specific perceptual elements of the experience, including the visual effects, narrative and narrator: "Amazing video! The apparition of Victoria at 4:48 gave me goosebumps! Well done!";

"I don't know what excites me more: Dietmar Wunder as speaker? These incredibly brilliant animations? Just fantastic!"; "I don't know what emotions the spokesman, the pictures, the animations and the writing triggered in me, but in the end I actually had to wipe away a tear. Incredibly great video and a brilliant experience!".

It is worth noting that in some comments there is an indication for viewers enduring involvement in the subject: "[..] this is probably also due to the emotions that I pretty much glorify the Romans and am a little fan boy :)"; "I've always been a fan of gladiators and this video is just so awesome and you can imagine everything much better now".

A virtual travel for an encounter with art can be experienced in Meeting Rembrandt $V R^{3}$ (Force Field VR, 2017), created in partnership with Rijksmuseum and Samsung Gear VR as part of Oculus VR experiences. The project was awarded at the Cinequest Film and VR Festival with Best Educational VR award 2018. In around 7 minutes, this VR film transports viewers back to 1642 in Amsterdam during the Golden Age to provide a face-to-face experience with the artist while he is giving life to his most famous painting, The Night Watch. Diffrently from other immersive experiences of paintings, the focus of the storytelling is not the painting itself, but the personal life of the artist (ViMM, 2020). The users can experience the creative process of the painter and get an understanding of why his work was so controversial in that age in an interactive way, with Rembrandt addressing them in first person and responding to their actions. The experience opens with the view of Rembrandt's house in Amsterdam in present days, stopping at the entrance of the Rembrandt's Huis Museum. The environment switches to older period of time and the user can see old buildings, before meeting the artist. The VR film recreates Rembrandt's house and surroundings, using live actors playing against a rich computer-generated environment.

From the analysis of comments, positive appreciation for the storytelling experience emerges in general: "Amazing. This is how stories need be told."; "Exceeded my expectation. As a frequent museum goer I didn't expect much but it was actually very enjoyable! Thank you to the creators of this great experience". Other users enjoyed specific elements, including the images, music and actors ("I really enjoyed the ambience of the art and music along with the actors and costumes. Highly recommended!").

Many viewers are enthusiasts particularly for the time travel ("Time-travel without leaving your home!") and the face-to-face encounter with the painter: "Totally amazing. You cannot help but feel you have met Rembrandt. This is an exceptional VR experience. Highly recommended". Strong emotional responses are also reported: "I mean this sincerely .... If someone were to ask me what is the ONE reason they should get into VR, I'd point to this app. Moved to tears by the trailer alone. Masterful!"

The experience was designed to be accessible to everyone, without sensors or controllers, and the importance of this aspect is confirmed in the analysis:

"I used this to show my Mom VR and so she could try something. She is a hemiplegic and since it only required her to look around, not use a remote or press buttons once I got it

\footnotetext{
${ }^{3}$ www.oculus.com/experiences/gear-vr/1297352360374984/
} 
started, she was able to do it. She smiled, turned her head, and made audible sounds. All of which are really rare."

Another relevant aspect concerns its potential for educational use, as many highlighted: "Wonderful way for learning about history of art."; "Past and present available with VR. No time machine needed, this experience is brilliant example of future theather and mite even history books replacement for future generation."

A totally different approach for bringing an art masterpiece to life through cinematic VR characterizes Dreams of Dali ${ }^{4}$ (Goodby Silverstein \& Partners, 2016), which takes viewers inside the painting Archeological Reminiscence of Millet's Angelus created by Salvador Dalì in 1935. This award-winning experience was created produced by the Dali Museum (St. Petersburg, Florida) as part of the exhibition Disney and Dali: Architects of the Imagination, and was released on the Oculus VR platform in 2018. It aims to take viewers inside the mind of the surrealist artist, bringing to life a CGI dreamscape, as a GS\&P creative director on the project explained the project: "We think Dalí imagined a real place and this painting was his way of expressing it." (Vr-reviews, 2016). Users can move around inside the surrealist landscape and listen what could be the artist's thoughts in his own voice. The imaginative VR scenario also incorporates some motifs from his other works in the museum's permanent collection.

The analysis of the many comments posted by viewers shows that the experience is successful in providing an immersion in the fantastic world of surrealism, which fuels viewers' imagination and the sense of stepping into Dali's mind "more intimately than before". According to the fans of his art, the unique nature of Dali visual world finds a perfect expression in VR ("It's like it was made for VR. Maybe Dali had a VR mind..."). The symbiosis between the medium and the art content is highlighted in relation to the new type of experience ("I completely did not expect anything like that - I've just experienced a whole new kind of experience I didn't even know I was missing."). Further, it appears to significantly impact the perception and understanding of the painter's work: "Truly amazing! It has forever changed the way I will look at the art of Dalí"; "if you never understood Dali.......after this magnificent piece of work, you will!!!”.

Several viewers asked for more contents, notably a longer experience, and also a videogame based on the elements of Dali's universe:

"SUGGESTION FOR THE GAME: a guy finds an artifact in a house where Salvador Dali himself lived. He takes this artifact home and activates it somehow. At night this artifact works on his dreams and somehow he gets connected to Dali's world which he finds out is actually a real parallel world. After some missions and exploration he finds out that Salvador and his wife Gala might not be dead, but found a way to live on in this parallel world."

Many expressed love for the visual aesthetics of the experience, although it had very different effects on viewers, from calming to mesmerizing and creepy feelings ("While visually interesting, this was one of the most creepy VR experiences I ever had. Extremely uncanny"). Emotional reactions reported by viewers included happiness, surprise, nostalgia, and even fear. For some viewers, it provoked an emotion that they cannot explain: "This causes an emotion I am not equipped to comprehend"; "I don't know what kind of

\footnotetext{
${ }^{4}$ https://thedali.org/exhibit/dreams-of-dali-in-virtual-reality/
} 
strings it touched in my heart, but somehow I was moved to the point of tears in my eyes." In other cases, strong emotional responses are reported also in relation to the physical sensation experienced in the virtual environment: "My heart is pounding out of my chest! What an experience! I am not afraid of heights in real life but this actually played with my emotions... I LOVE IT!".

Even viewers that are not engaged with Dali or Surrealism found the experience to be enjoyable and awesome, and it is considered "perfect to get in touch with art for the young people". As one young viewer commented, this experience can serve as a valuable first encounter with art: "My teacher showed me this and now I'm in love I love his art and I'm only 11 please make more videos like this". The experience evoked memories of personal visits to the museums and there are indications for a positive effect on intentions to visit the museum ("I have to visit The Dali museum in St. Petersburg, once it is possible again."; "Now I want to go to Petersburg").

The last project, The Man Behind Notre-Dame ${ }^{5}$ (TARGO, 2019) is a VR documentary set at present times offering an encounter with the man who guides the Cathedral in Paris, the Rector-Archpriest Patrick Chauvet, shedding light on his life, mission and what it means to be churchman in the modern age. This VR experience, coproduced by Channel Histoire (TF1 Group), was part of the competition section of VIRTUAL WORLDS at the Filmfest Munchen 2019 and has been featured as Best of VR by YouTube. The documentary is structured into three chapters and follows the daily life of the Rector-Archpriest, as he guides the audience in a behind-the-scenes journey of the cathedral. The experience takes the audience to different locations and contexts throughout the cathedral that are not accessible to the general public, including the sacristy, office, and the roof, which was damaged by the fire some months later. The story told in this VR experience is centred on the person of Chauvet who reveals his perspective on the significance of this cultural site. The storytelling approach centred on the protagonist through the first-person narration is appreciated ("the rector's view is intimate!"). The analysis of comments shows that the experience evoked emotions and reflections about the impact of the tragic fire that damaged the Cathedral in April 2019 ("It's a dramatic event, we cannot be indifferent."). Indeed, this experience offers some of the most recent immersive footage of the Cathedral before the tragic event, providing an historical documentation that was later included in another VR documentary created in 2020 by the same company "Rebuilding Notre Dame" to narrate the past, present and future of the Cathedral as shaped by this tragic event that has marked history.

\subsection{Discussion}

The exploratory analysis provides several insights into the key dimensions of storytelling considered in this study. It shows that storytellers have used this new medium to tell stories related to very different cultural heritage settings for educational, edutainment, accessibility and awareness purposes. The experiences are successful in targeting the wider audience at home though being equally useful to complement "real" onsite visits. They are found to be also used by teachers in their classes with positive reactions, confirming the relevance of digital storytelling for making the past more engaging for the young generations of digital natives (Pujol et al., 2012).

In the analyzed cases, cVR was used to create very different types of narrative experiences, from the travel in the past to the immersion into a masterpiece to the encounter with

\footnotetext{
5 https://www.targostories.com/cathedral-vr-targo
} 
protagonists of cultural heritage. However, in all the experiences an emphasis can be found on the people that revolve around heritage/exhibits and creating a link between them and viewers, in line with previous guidelines on emotive storytelling (Katifori et al., 2018; Perry et al., 2017). Even in the case of Dreams of Dali, where any human characters are present in the experience, the motivation of the storytellers was to provide an immersion into the painter's mind to reveal his unique perspective of art rather than explain the painting itself. Some experiences are more interactive and the users are placed at the very center of the scenes, being addressed by the characters. Interaction with the virtual environment is differently perceived, with users often expressing the desire for more interaction rather than just look around as well as for particular ways to explore the environment ("a VR flythrough would be fantastic."; "I think an automatic low-speed air ride would be better") or report problems with the audiovisual flow ("jumping from scene to scene is a little disorienting"). The perceived visual appeal emerges as an important aspect of the experience, in line with previous research (Marasco, 2017).

The analysis of users' comments highlights the range of emotional reactions elicited by the experiences, confirming previous research on the potential of this medium to arouse emotions (Durnell, 2018; Ding et al., 2018; Jones, 2017, 2019; Shin, 2018). However, the analysis indicates that emotional responses are provoked by different factors that need to be further explored for improving the design of immersive storytelling experiences. Indeed, emotional responses are found to be related to the surprising power of the VR as a ground breaking technology (Durnell, 2018), to the perceived novelty of the experience (Ding et al., 2018; Pujol-Tost, 2019), to the sense of presence in the virtual environment (Shin, 2018) and to specific elements of narrative immersion, such as voice over narrator, imagery and visual effects, actors and scenes. In some cases, the users reported strong responses that appear to be elicited by a mix of perceptual cues and narrative elements that they are not able to identify and explain themselves. This could be the effect of the "magic formula" as suggested by Roussou (2008), where all the elements of the immersive narrative tie to create a strong emotionally engaging experience. While previous studies explored the relationship between presence and narrative-related effects in immersive experiences (e.g., Diemer et al., 2015; Gorini et al., 2011; Shin, 2018), future research is needed to assess the higher emotional effect of narrative immersion in relation to other experiential elements and to storytellers' intentions. Further examination is also warranted to provide evidence supporting the relationship between emotional engagement and the memorability of the experience in line with Han et al. (2020) and Pagano et al. (2020).

Moreover, the findings provided evidence of a positive impact of the experience on viewers' cognitive and affective attitude towards the featured subject and also on intentions to visit the cultural site. In this regard, it would be necessary to further explore the impacts of narrative immersion on users' attitude and behaviour towards the cultural heritage subject. Current marketing research is indeed concerned to understand whether VR experiences decrease or fuel users' intentions to visit the featured cultural destinations (Guttentag, 2010; Deng et al., 2018; Lin et al., 2020; Marasco et al., 2018). In this direction, Li \& Chen (2019) distinguished the influence of VR contents from the effect of tourists' perception of the VR technology on visiting intentions. The recent review by Rach \& Scott (2020) has raised the need to fill an important gap in research on the use of VR in marketing by considering the effects of narrative components on presence and immersion in VR through narrative theory. Based on this perspective, future research could assess the effectiveness of these VR experiences for cultural tourism marketing by investigating the influence of narrative engagement on users' behavioral intentions towards the featured cultural heritage resources.

Finally, the analysis provides insights into the potential effect of users' engagement with the featured heritage on emotional responses. Many viewers explicitly explained their positive 
emotions in the light of their engagement with the subjects of the cinematic experiences. Previous research has demonstrated the role of prior knowledge in relation to the success of narrative transportation (Green, 2004) and the outcomes of storytelling (Selmanovic et al., 2018). Deng et al. (2018) have found a positive relationship between the enduring involvement of users with museum visits and intention to have an actual museum experience after the virtual museum experience, suggesting that VR experiences will induce users with high enduring involvement to consume the experience even more. By contrast, the study by Archer \& Finger (2018) in the context of immersive journalism has showed the highest empathetic response was registered in users who were unfamiliar with the stories they viewed. Therefore, more research is needed to assess the effect of these immersive narrative experiences in relation to their familiarity and cultural engagement in order to better target the market segments.

\section{CONCLUSIONS}

This study provided an explorative analysis of storytellers aims, narratives and storyreceivers reactions in cVR projects created in the specific context of cultural heritage. It is not without limitations. First, it considered only a small sample of projects and the analysis of other relevant experiences may alter the discussion of findings. Further, a varied total amount of user-generated data is available for the different cVR experiences considered in the analysis. Finally, it was not possible to ascertain whether all the viewers that commented the VR films experienced them with VR devices. For some projects, indeed, instructions are provided online to viewers on the use of three different display types (mobile phone, computer, VR device). Notwithstanding these limitations, the study offers several insights into the use of $\mathrm{cVR}$ for creating storytelling experiences in this context, included their potential to emotionally engage viewers. This is considered a key issue for the design of meaningful and memorable experiences based on VR in cultural heritage tourism (Han et al., 2020; Pagano et al., 2020; Roussou, 2008). As VR is increasingly adopted for cultural heritage storytelling and the cVR medium itself starts to find a place in this context, there is the opportunity to further explore its potential for the development of innovative cultural experiences beyond virtual tours. This opportunity is substantiated by the prospected growth of the market size of consumer VR in the next years and of audiovisual products consumed through online VR platforms.

At the time of this study, the relevance of the possibilities offered by VR for cultural experiences is more than ever highlighted due to the shut-in conditions of visitor economies deriving from the outbreak of the COVID-19 pandemic. The global coronavirus crisis has severely limited the full accessibility to museums and other cultural heritage sites for safeguarding the health and safety of their visitors and staff. In these conditions, digital channels and VR experiences have been promoted as unique vehicles to keep people connected to history and culture, enable cultural institutions to maintain and nurture the relationship with their audience and allow visitors to continue to travel across heritage destinations. It can be expected that these conditions will contribute to motivate unusual demand segments to embrace cultural virtual tourism as well as cultural institutions to invest in these innovative tools. Therefore, future research pursuing a closer dialogue between disciplinary perspectives is essential to advance knowledge and practice on the use of stories as a design tool for creating virtual experiences that contribute to greater sustainability of cultural heritage tourism.

\section{References}

Aman, S., \& Szpakowicz, S. (2007). Identifying expressions of emotion in text. Proceedings of the International Conference on Text, Speech and Dialogue (pp. 196-205). Springer. 
Archer, D., \& Finger, K. (2018). Walking in Another's Virtual Shoes: Do 360-Degree Video News Stories Generate Empathy in Viewers? Columbia Journalism School: Tow Center for Digital Journalism

Argyriou, L., Economou, D., \& Bouki, V. (2020). Design methodology for $360^{\circ}$ immersive video applications: the case study of a cultural heritage virtual tour. Personal and Ubiquitous Computing, 1-17. https://doi.org/10.1007/s00779-020-01373-8

Bedford, L. (2001). Storytelling: The real work of museums. Curator: the museum journal, 44(1), 2734.

Bekele, M. K., Pierdicca, R., Frontoni, E., Malinverni, E. S., \& Gain, J. (2018). A survey of augmented, virtual, and mixed reality for cultural heritage. Journal on Computing and Cultural Heritage, 11(2), $1-36$.

Brownlee, J. (2016, November 9). How the American Museum of Natural History is Spotlighting its Huge Unseen Collection. FastCompany.

Calvi, L., \& Hover, M. (2018). Becoming Vincent: A Multifaceted Story in a Multifaceted Ecosystem. In Museum Experience Design (pp. 279-300). Springer.

Carrozzino, M., \& Bergamasco, M. (2010). Beyond virtual museums: Experiencing immersive virtual reality in real museums. Journal of Cultural Heritage, 11(4), 452-458.

Champion, E.M. (2019, March 4). Virtual reality adds to tourism through touch, smell and real people's experiences. The Conversation.

Cho, J., Lee, T. H., Ogden, J., Stewart, A., Tsai, T. Y., Chen, J., \& Vituccio, R. (2016). Imago: presence and emotion in virtual reality. Proceedings of ACM SIGGRAPH 2016 VR Village (pp. 1-2).

Del Verme, L. (2019). Un itinerario 'ad patres'. Il cimitero delle Fontanelle a Napoli tra tecnologia e creatività. Archeologia e Calcolatori n. XXX, 405-421.

Demetrescu, E., Palombini, A., Pietroni, E., Forlani, M., \& Rufa, C. (2016). Making movies: nuove frontiere per la comunicazione audiovisiva del passato. Archeologia e Calcolatori, 8, 203-210

Deng, X., Unnava, H. R., \& Lee, H. (2019). “Too true to be good?” when virtual reality decreases interest in actual reality. Journal of Business Research, 100, 561-570.

Diemer, J., Alpers, G.W., Peperkorn, H.M., Shiban, Y., Mühlberger, A. (2015). The impact of perception and presence on emotional reactions: a review of research in virtual reality. Frontiers in Psychology 6, 26.

Ding, N., Zhou, W., \& Fung, A. Y. (2018). Emotional effect of cinematic VR compared with traditional 2D film. Telematics and Informatics, 35, 1572-1579

Dooley, K. (2017). Storytelling with virtual reality in 360-degrees: a new screen grammar. Studies in Australasian Cinema, 11(3), 161-171.

Douiji, Y., Mousannif, H., Moatassime, H. (2016). Using YouTube comments for text-based emotion recognition. Procedia Computer Science, 83, 292-299.

Durnell, L. A. (2018). Emotional Reaction of Experiencing Crisis in Virtual Reality (VR)/360 ${ }^{\circ}$. [Doctoral Dissertation, Fielding Graduate University ]. ProQuest Dissertations \& Theses Global.

Ekman, P. (1992). An Argument for Basic Emotions. Cognition and Emotion. 6, 169-200.

Gavalas, D., Sylaiou, S., Kasapakis, V., \& Dzardanova, E. (2020). Special issue on virtual and mixed reality in culture and heritage. Personal and Ubiquitous Computing, 1-2. https://doi.org/10.1007/s00779-020-01377-4

Gödde, M., Gabler, F., Siegmund, D., \& Braun, A. (2018). Cinematic narration in VR-Rethinking Film conventions for 360 degrees. Proccedings of the International Conference on Virtual, Augmented and Mixed Reality (pp. 184-201).

Gorini, A., Capideville, C. S., De Leo, G., Mantovani, F., \& Riva, G. (2011). The role of immersion and narrative in mediated presence: the virtual hospital experience. Cyberpsychology, Behavior, and Social Networking, 14(3), 99-105.

Green, M. C. (2004). Transportation into narrative worlds: The role of prior knowledge and perceived realism. Discourse processes, 38(2), 247-266.

Gutiérrez, M., Vexo, F., \& Thalmann, D. (2008). Stepping into virtual reality. Springer.

Guttentag, D. A. (2010). Virtual reality: Applications and implications for tourism. Tourism Management, 31(5), 637-651.

Han, D. I. D., Weber, J., Bastiaansen, M., Mitas, O., \& Lub, X. (2020). Blowing your mind: a conceptual framework of augmented reality and virtual reality enhanced cultural visitor experiences using EEG experience measures. International Journal of Technology Marketing, 14(1), 47-68. 
Jaunt (2018). The Cinematic VR Field Guide. Jaunt, Inc. https://creator.oculus.com/learn/cinematicvr-field-guide/

Jones, S. (2017). Disrupting the narrative: immersive journalism in virtual reality. Journal of Media Practice, 18(2-3), 171-185.

Jones, S. (2019). Towards the essence of cinematic VR: embracing new technologies to define a medium. In: tom Dieck, M.C. \& Jung, T. (Eds.). Augmented Reality and Virtual Reality. The power of $A R$ and $V R$ for Business (pp. 321-335), Springer Nature.

Katifori, A., Roussou, M., Perry, S., Drettakis, G., Vizcay, S., \& Philip, J. (2018). The EMOTIVE ProjectEmotive Virtual Cultural Experiences through Personalized Storytelling. Proceedings of CIRA@ EuroMed, 11-20.

Kim, M. J., Lee, C. K., \& Jung, T. (2020). Exploring consumer behavior in virtual reality tourism using an extended stimulus-organism-response model. Journal of Travel Research, 59(1), 69-89.

Kubitzek, B. (2019). Cognition and Emotion in Cinematic Virtual Reality: What are the challenges in production to creating an emotional response? [Thesis, Karlstad University]. Diva Portal.

Li, T., \& Chen, Y. (2019). Will virtual reality be a double-edged sword? Exploring the moderation effects of the expected enjoyment of a destination on travel intention. Journal of Destination Marketing \& Management, 12, 15-26.

Lin, L. P. L., Huang, S. C. L., \& Ho, Y. C. (2020). Could virtual reality effectively market slow travel in a heritage destination? Tourism Management, 78, 104027.

Mateer, J. (2017). Directing for Cinematic Virtual Reality: how the traditional film director's craft applies to immersive environments and notions of presence. Journal of Media Practice, 18(1), 1425.

Marasco A. (2017), Innovare l'esperienza di fruizione del patrimonio culturale: il modello \#VIAGGIARTE, in: Del Verme, Arrigo (Eds.). Innovazione della fruizione: le nuove frontiere della digitalizzazione. Atti della Giornata di studio (Caiazzo) (pp. 59-63), Edizioni San Bonaventura Onlus.

Marasco, A., Buonincontri, P., van Niekerk, M., Orlowski, M., \& Okumus, F. (2018). Exploring the role of next-generation virtual technologies in destination marketing. Journal of Destination Marketing \& Management, 9, 138-148.

Min-sik, Y. (2018, June 21). Korea's cultural heritage brought to life via VR. The Korea Herald.

Montagud, M., Orero, P. \& Matamala, A. (2020). Culture 4 all: accessibility-enabled cultural experiences through immersive VR360 content. Personal and Ubiquitous Computing, 1-2. https://doi.org/10.1007/s00779-019-01357-3

Pagano, A., Palombini, A., Bozzelli, G., Nino, M. D., Cerato, I., \& Ricciardi, S. (2020). ArkaeVision VR Game: User Experience Research between Real and Virtual Paestum. Applied Sciences, 10(9), 3182.

Palombini, A. (2012). Narrazione e virtualità: possibili prospettive per la comunicazione museale. DigItalia, 1, 9-22.

Palombini, A. (2017). Storytelling and telling history. Towards a grammar of narratives for Cultural Heritage dissemination in the Digital Era. Journal of cultural heritage, 24, 134-139.

Perry, S., Roussou, M., Economou, M., Young, H., \& Pujol, L. (2017). Moving Beyond the Virtual Museum: Engaging Visitors Emotionally. Proceedings of 23rd Int'l Conference on Virtual Systems and Multimedia -VSMM 2017. Dublin, Ireland: IEEE

Pietroni, E. (2019). Experience Design, Virtual Reality and Media Hybridization for the Digital Communication Inside Museums. Applied System Innovation, 2(4), 35.

Pillai, J. S., \& Verma, M. (2019). Grammar of VR Storytelling: Narrative Immersion and Experiential Fidelity in VR Cinema. Proceedings of the 17th International Conference on Virtual Reality Continuum and its Applications in Industry (pp. 1-6) VRCAI '19, November 14-16, Brisbane, QLD, Australia

Pujol, L., Roussou, M., Poulou, S., Balet, O., Vayanou, M., \& Ioannidis, Y. (2012). Personalizing interactive digital storytelling in archaeological museums: the CHESS project. Proceedings of 40th annual conference of computer applications and quantitative methods in archaeology. Amsterdam University Press.

Pujol-Tost, L. (2018). Cultural presence in virtual archaeology: An exploratory analysis of factors. Presence: Teleoperators and Virtual Environments, 26(03), 247-263. 
Rach, M., \& Scott, R. (2020). The Use of Virtual Reality in Marketing: Exploring the Need for Technology and Language Adaptation to Create High Quality Immersive Experiences. In Marketing and Smart Technologies (pp. 67-77). Springer.

Reyes, M. C. (2018,). Measuring User Experience on Interactive Fiction in Cinematic Virtual Reality. In International Conference on Interactive Digital Storytelling (pp. 295-307). Springer, Cham.

Rizvic, S., Okanovic, V., \& Boskovic, D. (2020). Digital Storytelling. In Visual Computing for Cultural Heritage (pp. 347-367). Springer.

Roussou, M. (2002). Virtual heritage: from the research lab to the broad public. Bar International Series, 1075, 93-100.

Roussou, M. (2008). The components of engagement in virtual heritage environments. Proceedings of New Heritage: Beyond Verisimilitude - Conference on Cultural Heritage and New Media, Hong Kong, pp. 265-283.

Roussou, M., Ripanti, F., \& Servi, K. (2017). Engaging visitors of archaeological sites through 'emotive'storytelling experiences: a pilot at the Ancient Agora of Athens. Archeologia $e$ Calcolatori, 28(2), 405-420.

Schofield, G., Beale, G., Smith, N., Fell, M., Hook, J., Hadley, D., Murphy, D., Richards, J. and Thresh, L. (2018). Viking VR: Designing a Virtual Reality Experience for a Museum. Proceedings of Designing Interactive Systems Conference 2018 (DIS '18), Hong Kong (pp. 805-815).

Selmanovic E., Rizvic S., Harvey C., Boškovic D., Hulusic V., Chahin M., Šljivo S. (2018). VR video storytelling for intangible cultural heritage preservation. Proceedings of 16th EUROGRAPHICS workshop on graphics and cultural heritage.

Shin, D. (2018). Empathy and embodied experience in virtual environment: To what extent can virtual reality stimulate empathy and embodied experience?. Computers in Human Behavior, 78, 64-73.

Sylaiou, S., \& Dafiotis, P. (2020). Storytelling in Virtual Museums: Engaging A Multitude of Voices. In Visual Computing for Cultural Heritage (pp. 369-388). Springer.

Tussyadiah, I. P., Wang, D., Jung, T. H., \& tom Dieck, M. C. (2018). Virtual reality, presence, and attitude change: Empirical evidence from tourism. Tourism Management, 66, 140-154.

ViMMPlus (2020). Meeting Rembrandt: Master of reality. Virtual Multimodal Museums Plus.

Weissenfeld, K., Abramova, 0., \& Krasnova, H. (2017). Understanding storytelling in the context of information systems. Proceedings of 22nd Americas Conference on Information Systems (AMCIS 2017), Boston, USA. 